\title{
Airborne FDA-MIMO Radar Modeling and Detection Performance Analysis
}

\author{
Yisheng YAN ${ }^{1}$, Wen-Qin WANG ${ }^{2}$, Abdul BASIT ${ }^{2}$, Jingye CAI ${ }^{1}$ \\ ${ }^{1}$ School of Information and Software Engineering, University of Electronic Science and Technology of China, \\ Chengdu, 611731, P. R. China \\ ${ }^{2}$ School of Information and Communication Engineering, University of Electronic Science and Technology of China, \\ Chengdu, 611731, P. R. China
}

yishengyan0308@gmail.com,wqwang@uestc.edu.cn, abdulbasit@iiu.edu.pk, jycai@uestc.edu.cn

Submitted September 30, 2020 / Accepted March 13, 2021

\begin{abstract}
In the conventional frequency diverse array (FDA) radar designs, generalized likelihood ratio test (GLRT) detection utilizes coherent pulses. However, the impacts of an FDA multiple-input multiple-output (FDAMIMO) radar system for detection with incoherent pulses have not been systematically investigated. In this paper, we present an incoherent square-law detector to analyse the performance of both the coherent and non-coherent airborne FDA-MIMO radars in a Neyman-Pearson sense. Moreover, the closed-form expressions of an incoherent square-law detector for the FDA-MIMO radars are derived. For a coherent FDA-MIMO radar, the optimal performance is achieved at a high signal-to-noise ratio (SNR), whereas the superiority of a non-coherent FDA-MIMO radar in distinguishing range dependent targets is validated. The corresponding theoretical derivations are verified by the extensive numerical results to show an improved performance.
\end{abstract}

\section{Keywords}

Coherent and non-coherent FDA-MIMO radar, airborne radar, square-law detector, incoherent pulses, NeymanPearson sense, detection performance, range dependent targets

\section{Introduction}

Array-signal processing (ASP) for phased-array (PA) and multiple-input multiple-output (MIMO) radars have been extensively investigated for various applications [1-4]. However, there is always a room for improvement. For instance, if the radar cross section (RCS) is fluctuated, the performance degradation has not been well handled. Although waveform diversity can be employed to mitigate the angular spread [5], [6], yet the degradation in its detection performance cannot be avoided. To improve detection performance, statistical MIMO radar utilizes a generalized likelihood ratio test (GLRT) detector with space-time coding configuration [7], but the required time/frequency synchronization remains a technical challenges [8]. Additionally, generalized constrained adaptive beamforming [9] has been used to suppress the interferences and range-ambiguous clutter. However, the extra training data are required for the conventional MIMO radars [10].

Contrary to the traditional PA, MIMO and frequency stepped techniques, frequency diverse array (FDA) uses a small frequency increment across its array elements [11-14] that helps to produce an angle, range and time dependent transmit beampattern to provide extra degrees of freedom (DOFs) in range and time dimensions [15-17]. Moreover, a secondary range dependence compensation (SRDC) method was proposed to alleviate range-ambiguous clutter [18], [19], where the extra DOFs of an FDA radar were exploited to suppress the interference and clutter [20,21] for an improved detection performance. Besides, time variance of an FDA radar plays an essential role in its applications that has been explored in the few recent literatures [22], [23].

Note that, the aforementioned literatures for FDAMIMO radar based detection concentrate on multiple coherent pulses [24], whereas a low complexity adaptive GLRT detector for an FDA radar was proposed in [25] to improve the detection probability of the range-dependent targets. Furthermore, a GLRT-based adaptive detector was proposed to enhance the detection performance of an FDA-MIMO radar [26], where an FDA combined with MIMO technique is used to mitigate the range ambiguity.

In this paper, we focus on deriving a closed form mathematical expression for the incoherent square-law detector based signal models of the airborne coherent and noncoherent FDA-MIMO radars. Compared with PA and MIMO radars, the multiple incoherent pulses based received echoes of the airborne FDA-MIMO radars are jointly space-timerange (STR) dependent, therefore the extra DOFs can be used to distinguish the range dependent targets. In addition, we present performance analysis of the airborne FDA-MIMO radar, especially, for different frequency increment configu- 
rations and distribution of antenna elements. The superiority of non-coherent FDA-MIMO radar in distinguishing range dependent targets is validated by extensive numerical results. In summary, our main contributions are listed as follows:

- We derive the jointly space-time-range (STR) dependent signal model for a generalize airborne FDA-MIMO radar, and analyse the influence of the frequency increment configurations on the reflection coefficients of an FDA-MIMO radar.

- Closed-form expressions are derived for the incoherent square-law detector of the coherent and non-coherent airborne FDA-MIMO radars in a Neyman-Pearson sense. The improvement of the detection performance for the incoherent pulses model of an FDA-MIMO radar is confirmed by theoretical derivations.

- Numerical examples are presented to compare the performance of the proposed coherent and non-coherent FDA-MIMO radars with traditional PA and MIMO. The improved target detection superiority of coherent FDA-MIMO at high SNR and improved distinguishing performance of range-dependent targets using noncoherent FDA-MIMO radar are verified by extensive numerical results.

The remaining sections are organized as follows. Section 2 formulates the received signal model of a general airborne FDA-MIMO radar. Section 3 presents the derivation of the square-law detector of both the coherent and non-coherent FDA-MIMO radars in Neyman-Pearson sense. Finally, simulation results are provided in Sec. 4 and conclusions are drawn in Sec. 5.

\section{Airborne FDA-MIMO Radar Signal Model}

Consider the FDA-MIMO radar with collocated $M$-element transmit array and $N$-element receive array. The carrier frequency of the $m$ th transmit element is expressed as

$$
f_{m}=f_{\mathrm{c}}+\Delta f_{m}, \quad m=1,2, \ldots, M
$$

where $\Delta f_{m}$ stands for the $m$ th frequency increment as compared to the reference carrier $f_{\mathrm{c}}$. Let $s_{m}(t)$ be the baseband signal transmitted by the $m$ th element, the $m$ th transmitted signal can be expressed as

$$
s_{m}(t) \exp \left(\mathrm{j} 2 \pi f_{m} t\right), \quad 0 \leq t \leq T_{\mathrm{s}}
$$

where $T_{\mathrm{S}}$ denotes the pulse duration.

Suppose there is a scatterer located at $\mathbf{P}$, as shown in Fig. 1 that is moving towards the radar platform with a radial velocity $v_{\mathrm{p}}$. Since the signal departs from the $m$ th transmit element, reflects off the target and returns to the $n$th receive element, the received signal can be expressed as

$$
y_{n m}(t)=\alpha_{n m} s_{m}\left(t-\tau_{n m}(t)\right) \exp \left(\mathrm{j} 2 \pi f_{m}\left(t-\tau_{n m}(t)\right)\right)
$$

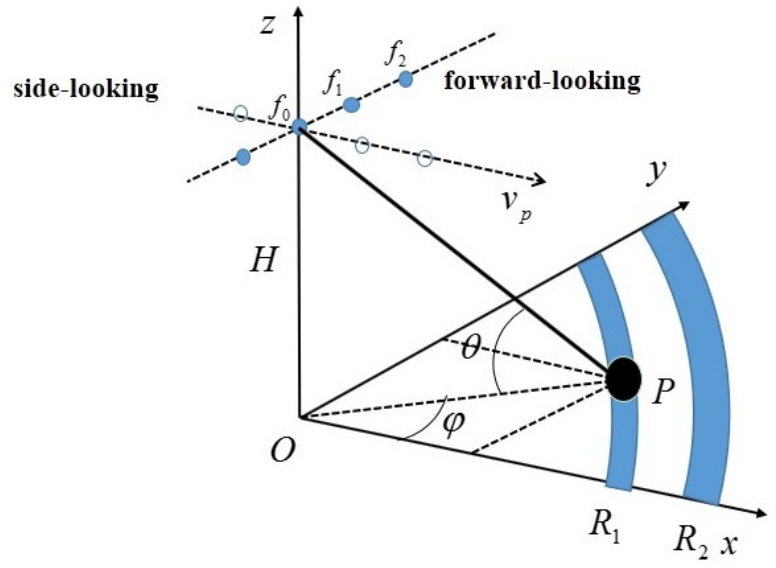

Fig. 1. Geometry of airborne linear FDA radar.

where $\alpha_{n m}$ is the reflection coefficient of the $n m$-th corresponding path and $\tau_{n m}(t)$ denotes the corresponding propagation delay. Following the convention in moving target detection, $s(t)$ is assumed to be composed of a train of incoherent pulses, as follow:

$$
s(t)=\sum_{l=0}^{L-1} \Phi(t-l T)
$$

where $L$ is the number of pulses, $\Phi(t), \quad 0 \leq t \leq T_{\mathrm{p}}$ is the baseband pulse signal with pulse duration $T_{\mathrm{p}}$, and $T$ is the pulse repetition interval (PRI).

Suppose the $m$ th transmit element and the $n$th receive element are located at the coordinates $\left(x_{m}, y_{m}, z_{m}\right)$ and $\left(x_{n}, y_{n}, z_{n}\right)$, respectively, the receiver needs a set of matched filters to separate the orthogonal transmitting signals in the $N$ receive elements. Therefore, we can get $N \times M \times L$ independent target echoes. Under the narrow-band and far-field assumptions, the slow-time echo sample of $y_{n m}(t)$ during the $l$ th PRI can be expressed as [22], [27]

$$
y_{n m}^{\mathrm{F}-\mathrm{M}}[l]=\alpha_{n m l} a_{n m}(\varphi, \theta) \omega_{l}(\varphi, \theta) e_{m l}(\Delta f) d_{m}\left(\Delta f, \Delta \tau_{\mathrm{s}}\right)
$$

where $\alpha_{n m l}$ is the complex reflection coefficient of $n m$-th path corresponding to the $l$-th incoherent pulse. $\varphi$ and $\theta$ are the elevation and azimuth angles of target to the airborne FDA-MIMO radar, respectively. Moreover, $\Delta \tau_{\mathrm{s}}=\tau-\tau_{\mathrm{s}}$ is the relative delay of target to the radar phase center, and the rest of the parameters are defined as

$$
\begin{aligned}
& a_{n}(\varphi, \theta)=\exp \left(\mathrm{j} \frac{2 \pi f_{\mathrm{c}}}{c}\left(\left[x_{n} \cos \varphi+y_{n} \sin \varphi\right] \cos \theta-z_{n} \sin \theta\right)\right), \\
& a_{m}(\varphi, \theta)=\exp \left(\mathrm{j} \frac{2 \pi f_{\mathrm{c}}}{c}\left(\left[x_{m} \cos \varphi+y_{m} \sin \varphi\right] \cos \theta-z_{m} \sin \theta\right)\right),
\end{aligned}
$$

$a_{n m}(\varphi, \theta)=a_{n}(\varphi, \theta) \otimes a_{m}(\varphi, \theta)$,

$\omega_{l}(\varphi, \theta)=\exp \left(-\mathrm{j} \frac{2 \pi f_{\mathrm{c}}}{c} 2 v_{\mathrm{p}} l T \cos \varphi \cos \theta\right)$,

$e_{m l}(\Delta f)=\exp (\mathrm{j} 2 \pi(m-1) \Delta f l T)$,

$d_{m}\left(\Delta f, \Delta \tau_{\mathrm{s}}\right)=\exp \left(\mathrm{j} 2 \pi(m-1) \Delta f \Delta \tau_{\mathrm{s}}\right)$ 
where $\otimes$ denotes the Kronecker product operator. According to [28], the reflection coefficient can be represented by

$$
\alpha_{n m l}=\alpha(n, l, \varphi, \theta, r)=\eta(n, \varphi, \theta, r) \rho(l, \varphi, \theta)
$$

where $\eta(n, \varphi, \theta, r)=A_{\mathrm{s}} G_{\mathrm{T}}(\varphi, \theta) L(r) G_{\mathrm{R}}(\varphi, \theta) B(n, \varphi, \theta)$ can be determined by the actual FDA-MIMO radar system, $L(r)$ stands for the reflectivity of the scatter, $G_{\mathrm{T}}(\varphi, \theta)$ and $G_{\mathrm{R}}(\varphi, \theta)$ are the transmit and receive beampatterns, respectively. Moreover, $B(n, \varphi, \theta)$ is the amplitude and phase relation for the receiver. Thus, the statistical characteristics of reflection coefficients for coherent and non-coherent FDA-MIMO radars can be expressed as

$$
\begin{gathered}
E\left[\alpha_{n m l} \alpha_{n^{\prime} m^{\prime} l^{\prime}}^{*}\right]=E\left[\alpha(n, l, \varphi, \theta, r) \alpha^{*}\left(n^{\prime}, l^{\prime}, \varphi^{\prime}, \theta^{\prime}, r^{\prime}\right)\right] \\
=\left|A_{\mathrm{s}}\right|^{2}\left|G_{\mathrm{T}}(\varphi, \theta)\right|^{2}\left|G_{\mathrm{R}}(\varphi, \theta)\right|^{2}|L(r)|^{2} \sigma(\varphi, \theta) \tilde{\rho}_{n n^{\prime}} \breve{\rho}_{l l^{\prime}}
\end{gathered}
$$

where $E[\cdot]$ is the expectation operator, and the amplitude of signal can be defined as $A_{\mathrm{s}}$, furthermore, $\tilde{\rho}_{n n^{\prime}}$ and $\breve{\rho}_{l l^{\prime}}$ denote the spatial decorrelation and time decorrelation, respectively. With the assumption of uniform target and the same shape of the scatterer spectrum corresponding to different azimuth and elevation angles, only the constant factor $\sigma(\varphi, \theta)$ is different.

Compared to the traditional PA and MIMO radars, the reflection coefficient of FDA-MIMO radars depend on its frequency increment $\Delta f$ and the distribution of antenna elements that are summarized in Tab. 1. According to the Swerling-II model, $\alpha_{n m l}$ can be regarded as a zero mean normal random variable with variance $\sigma_{\alpha}^{2}$. Thus the slowtime echo sample of coherent and non-coherent FDA-MIMO radars can be expressed as (9a) and (9b), respectively

$$
\begin{aligned}
y_{n m l}^{\mathrm{F}} & =\alpha a_{n m}(\varphi, \theta) \omega_{l}(\varphi, \theta) e_{m l}(\Delta f) d_{m}\left(\Delta f, \Delta \tau_{\mathrm{s}}\right), \\
y_{n m l}^{\mathrm{F}-\mathrm{M}} & =\alpha_{n m l} a_{n m}(\varphi, \theta) \omega_{l}(\varphi, \theta) e_{m l}(\Delta f) d_{m}\left(\Delta f, \Delta \tau_{\mathrm{s}}\right) .
\end{aligned}
$$

\section{Airborne FDA-MIMO Radar Target Detection}

\subsection{Incoherent Square-Law Detector}

Under the signal-plus-noise hypothesis, the $m$ th slow time sampling signal received by the $n$th receive antenna can be expressed as

$$
r_{n m}^{\mathrm{F}-\mathrm{M}}[l]=y_{n m}^{\mathrm{F}-\mathrm{M}}[l]+n_{n m}[l] .
$$

The receiver needs a set of matched filters to separate the orthogonal transmitting signals on the $N$ receive elements, so we can get $N \times M \times L$ independent target echoes. For notation convenience, $r_{n m}^{\mathrm{F}-\mathrm{M}}[l], y_{n m}^{\mathrm{F}-\mathrm{M}}[l]$ and $n_{n m}[l]$ can be rewritten as $r_{n m l}^{\mathrm{F}-\mathrm{M}}, y_{n m l}^{\mathrm{F}-\mathrm{M}}$ and $n_{n m l}$, respectively. Thus, the radar detection problem is formulated as follows

$$
\begin{aligned}
& H_{0} \text { : Target does not exits at detection unit, } \\
& H_{1} \text { : Target exits at detection unit. }
\end{aligned}
$$

In both of the hypotheses showed in (11), the received sampling signal can be expressed as

$$
r_{n m l}^{\mathrm{F}-\mathrm{M}}=\left\{\begin{array}{cc}
n_{n m l} & H_{0}, \\
y_{n m l}^{\mathrm{F}-\mathrm{M}}+n_{n m l} & H_{1} .
\end{array}\right.
$$

The $N \times M \times L$ independent sampling signals are given by

$$
\mathbf{r}^{\mathrm{F}-\mathrm{M}}=\left[r_{111}^{\mathrm{F}-\mathrm{M}}, \ldots, r_{11 \mathrm{~L}}^{\mathrm{F}-\mathrm{M}}, \ldots, r_{\mathrm{NM} 1}^{\mathrm{F}-\mathrm{M}}, \ldots, r_{\mathrm{NML}}^{\mathrm{F}-\mathrm{M}}\right]^{\mathrm{T}}
$$

where $\mathbf{r}^{\mathrm{F}-\mathrm{M}} \sim \mathcal{C N}\left(0, \sigma_{r}^{2} \mathbf{I}_{\mathrm{NML}}\right)$, and $\sigma_{r}^{2}$ denotes the variance of $r_{n m l}^{\mathrm{F}-\mathrm{M}}$. Moreover, the real part $\operatorname{Re}\left\{\mathbf{r}^{\mathrm{F}-\mathrm{M}}\right\} \sim$ $C \mathcal{N}\left(0, \frac{\sigma_{r}^{2}}{2} \mathbf{I}_{\mathrm{NML}}\right)$ is independent from the imaginary part $\operatorname{Im}\left\{\mathbf{r}^{\mathrm{F}-\mathrm{M}}\right\} \sim \mathcal{C N}\left(0, \frac{\sigma_{r}^{2}}{2} \mathbf{I}_{\mathrm{NML}}\right)$.

This paper uses the conventional square-law incoherent receiver (depicted in Fig. 2). Accordingly, the output vector of detector can be expressed as

$$
\mathbf{Z}^{\mathrm{F}-\mathrm{M}}=\left[z_{111}^{\mathrm{F}-\mathrm{M}}, \ldots, z_{11 \mathrm{~L}}^{\mathrm{F}-\mathrm{M}}, \ldots, z_{\mathrm{NM} 1}^{\mathrm{F}-\mathrm{M}}, \ldots, z_{\mathrm{NML}}^{\mathrm{F}-\mathrm{M}}\right]^{\mathrm{T}}
$$

where $z_{n m l}^{\mathrm{F}-\mathrm{M}}=\left|r_{n m l}^{\mathrm{F}-\mathrm{M}}\right|^{2},(n=1, \ldots, N ; m=1, \ldots M ; l=$ $1 \ldots, L)$, and $|\cdot|$ denotes the modulo operator. Therefore, $\mathbf{Z}^{\mathrm{F}-\mathrm{M}}$ is a Chi-square random variable of $2 \mathrm{NML}$ DOFs with the parameter $1 / \sigma_{n}^{2}$ under the null hypothesis $H_{0}$, and $1 /\left(\sigma_{\mathrm{F}-\mathrm{M}}^{2}+\sigma_{n}^{2}\right)$ under the alternate hypothesis $H_{1}$, where $\sigma_{n}^{2}$ and $\sigma_{\mathrm{F}-\mathrm{M}}^{2}$ are the variance of noise $n_{n m l}$ and signal $y_{n m l}^{\mathrm{F}-\mathrm{M}}$, respectively.

This paper derives the detection performance of coherent and non-coherent FDA-MIMO radars in next subsections, respectively.

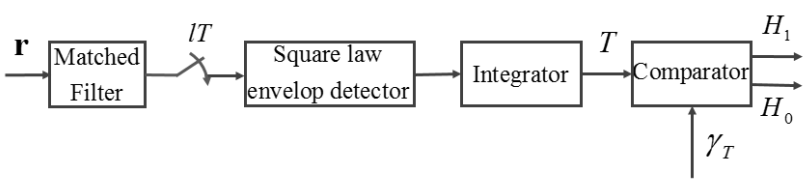

Fig. 2. Conventional incoherent square-law receiver.

\begin{tabular}{|c|c|c|c|c|}
\hline Reflection Coefficient & Statistical Covariance & Value of $\Delta f$ & Array Distribution & Array Structure \\
\hline Coherent model & $E\left[\alpha_{n m} \alpha_{n^{\prime} m^{\prime}}^{*}\right]=1, \forall n, m$ & $\Delta f \ll B_{\mathrm{s}}$ & Co-located & PA, coherent FDA-MIMO \\
\hline Independent model & $E\left[\alpha_{n m} \alpha_{n^{\prime} m^{\prime}}^{*}\right]=0, \forall n \neq n^{\prime}$ or $m \neq m^{\prime}$ & $\Delta f \approx B_{\mathrm{s}}$ & Widely Separated & MIMO, non-coh. FDA-MIMO \\
\hline Correlated model & $E\left[\alpha_{n m} \alpha_{n^{\prime} m^{\prime}}^{*}\right] \in(0,1), \forall n \neq n^{\prime}$ or $m \neq m^{\prime}$ & $0<\Delta f<B_{\mathrm{s}}$ & Co-located & General FDA \\
\hline
\end{tabular}

Tab. 1. Reflection coefficient model of FDA-MIMO radars. 


\subsection{Non-coherent FDA-MIMO Radar}

When the antennas are widely separated, the attenuation factor can be defined as $\delta_{n m}=1 /\left(d_{\mathrm{t} m} \cdot d_{\mathrm{r} n}\right)$, where $d_{\mathrm{t} m}$ and $d_{\mathrm{r} n}$ denote the distance from scatterers to the $m$ th transmit and $n$th receive antennas, respectively. Assuming the reflection coefficients of target are independent from noise, it is easy to verify that the probability density function (PDF) of $\mathbf{Z}^{\mathrm{F}-\mathrm{M}}$ is distributed as follows:

$$
\left\{\begin{array}{l}
p\left(\mathbf{Z}^{\mathrm{F}-\mathrm{M}} \mid H_{0}\right)=\prod_{l=1}^{L} \prod_{n=1}^{N} \prod_{m=1}^{M}\left(\frac{1}{\sigma_{n}^{2}} \exp \left(\frac{-z_{n m l}^{\mathrm{F}-\mathrm{M}}}{\sigma_{n}^{2}}\right)\right) \\
p\left(\mathbf{Z}^{\mathrm{F}-\mathrm{M}} \mid H_{1}\right)=\prod_{l=1}^{L} \prod_{n=1}^{N} \prod_{m=1}^{M}\left(\frac{1}{\sigma_{\mathrm{F}-\mathrm{M}}^{2}+\sigma_{n}^{2}} \exp \left(\frac{-z_{n m l}^{\mathrm{F}-\mathrm{M}}}{\sigma_{\mathrm{F}-\mathrm{M}}^{2}+\sigma_{n}^{2}}\right)\right)
\end{array}\right.
$$

Consequently, the likelihood ratio function can be calculated as

$$
\begin{aligned}
& L\left(\mathbf{Z}^{\mathrm{F}-\mathrm{M}}\right)=\frac{p\left(\mathbf{Z}^{\mathrm{F}-\mathrm{M}} \mid H_{1}\right)}{p\left(\mathbf{Z}^{\mathrm{F}-\mathrm{M}} \mid H_{0}\right)}= \\
& \prod_{l=1}^{L} \prod_{n=1}^{N} \prod_{m=1}^{M}\left(\frac{1}{\frac{\sigma_{\mathrm{F}-\mathrm{M}}^{2}}{\sigma_{n}^{2}}+1} \exp \left(\frac{z_{n m l}^{\mathrm{F}-\mathrm{M}}\left(\frac{\sigma_{\mathrm{F}-\mathrm{M}}^{2}}{\sigma_{n}^{2}}\right)}{\sigma_{n}^{2}\left(\frac{\sigma_{\mathrm{F}-\mathrm{M}}^{2}}{\sigma_{n}^{2}}+1\right)}\right)\right) .
\end{aligned}
$$

For derivation convenience, $\sigma_{\mathrm{F}-\mathrm{M}}^{2} / \sigma_{n}^{2}$ in (16) can be redefined as

$$
\frac{\sigma_{\mathrm{F}-\mathrm{M}}^{2}}{\sigma_{n}^{2}}=\frac{\delta_{n m}^{2} \delta_{\varphi \theta}^{2} \xi_{\Delta f}^{2} \sigma_{\alpha}^{2}}{\sigma_{n}^{2}}=\xi_{\mathrm{F}-\mathrm{M}}^{2} \zeta
$$

where $\xi_{\mathrm{F}-\mathrm{M}}^{2}=\delta_{n m}^{2} \delta_{\varphi \theta}^{2} \xi_{\Delta f}^{2}$ represents the unique parameter of non-coherent FDA-MIMO radar. Additionally, the geometric distance of the scatterers and factor corresponding to frequency increment can be defined as $\delta_{\varphi \theta}^{2}$ and $\xi_{\Delta f}^{2}$, respectively. Moreover, $\zeta=\mathrm{SNR}_{0}=\sigma_{\alpha}^{2} / \sigma_{n}^{2}$ denotes the inherent SNR of the radar system, where $\sigma_{\alpha}^{2}$ represents the variance of the reflection coefficients. Thus, (16) can be rewritten as

$$
\begin{aligned}
& L\left(\mathbf{Z}^{\mathrm{F}-\mathrm{M}}\right)=\frac{p\left(\mathbf{Z}^{\mathrm{F}-\mathrm{M}} \mid H_{1}\right)}{p\left(\mathbf{Z}^{\mathrm{F}-\mathrm{M}} \mid H_{0}\right)}= \\
& \prod_{l=1}^{L} \prod_{n=1}^{N} \prod_{m=1}^{M}\left(\frac{1}{\xi_{\mathrm{F}-\mathrm{M}}^{2} \zeta+1} \exp \left(\frac{z_{n m l}^{\mathrm{F}-\mathrm{M}} \xi_{\mathrm{F}-\mathrm{M}}^{2} \zeta}{\sigma_{n}^{2}\left(\xi_{\mathrm{F}-\mathrm{M}}^{2} \zeta+1\right)}\right)\right) .
\end{aligned}
$$

Applying the logarithm operation on both sides of (18), the log-likelihood ratio can be obtained as

$$
\begin{aligned}
& \ln \left(L\left(\mathbf{Z}^{\mathrm{F}-\mathrm{M}}\right)\right)= \\
& \sum_{l=1}^{L} \sum_{n=1}^{N} \sum_{m=1}^{M}\left(\ln \left(\frac{1}{\xi_{\mathrm{F}-\mathrm{M}}^{2} \zeta+1}\right)+\left(\frac{z_{n m l}^{\mathrm{F}-\mathrm{M}} \xi_{\mathrm{F}-\mathrm{M}}^{2} \zeta}{\sigma_{n}^{2}\left(\xi_{\mathrm{F}-\mathrm{M}}^{2} \zeta+1\right)}\right)\right) .
\end{aligned}
$$

For notational simplicity, we define $\Upsilon_{n m l}^{\mathrm{F}-\mathrm{M}}=z_{n m l}^{\mathrm{F}-\mathrm{M}} / \sigma_{n}^{2}$ and $\kappa_{n m l}^{\mathrm{F}-\mathrm{M}}=\xi_{\mathrm{F}-\mathrm{M}}^{2} \zeta /\left(\xi_{\mathrm{F}-\mathrm{M}}^{2} \zeta+1\right)$. Accordingly, (19) can be simplified to

$$
\ln \left(L\left(\mathbf{Z}^{\mathrm{F}-\mathrm{M}}\right)\right)=\sum_{l=1}^{L} \sum_{n=1}^{N} \sum_{m=1}^{M}\left(\ln \left(\frac{1}{\xi_{\mathrm{F}-\mathrm{M}}^{2} \zeta+1}\right)+\kappa_{n m l}^{\mathrm{F}-\mathrm{M}} \Upsilon_{n m l}^{\mathrm{F}-\mathrm{M}}\right)
$$

According to [29], the corresponding detector structure of non-coherent FDA-MIMO radar is

$$
T_{\mathrm{F}-\mathrm{M}}^{\mathrm{I}}=\sum_{l=1}^{L} \sum_{n=1}^{N} \sum_{m=1}^{M}\left(\kappa_{n m l}^{\mathrm{F}-\mathrm{M}} \Upsilon_{n m l}^{\mathrm{F}-\mathrm{M}}\right)_{\mathrm{H}_{0}}^{\stackrel{H_{1}}{>}} \eta
$$

where $T_{\mathrm{F}-\mathrm{M}}^{\mathrm{I}}$ denotes the detection statistics of non-coherent FDA-MIMO radar, and $\eta$ is the threshold determined by the probability of false alarm.

$$
\begin{aligned}
& \alpha_{0}^{\mathrm{I}}=\frac{\left(\sum_{l=1}^{L} \sum_{n=1}^{N} \sum_{m=1}^{M} \tilde{\kappa}_{n m l}^{\mathrm{F}-\mathrm{M}}\right)^{2}}{\sum_{l=1}^{L} \sum_{n=1}^{N} \sum_{m=1}^{M}\left(\tilde{\kappa}_{n m l}^{\mathrm{F}-\mathrm{M}}\right)^{2}}=\frac{\left(\sum_{l=1}^{L} \sum_{n=1}^{N} \sum_{m=1}^{M} \frac{\xi_{\mathrm{F}-\mathrm{M}}^{2} \zeta}{2\left(\xi_{\mathrm{F}-\mathrm{M}}^{2} \zeta+1\right)}\right)^{2}}{\sum_{l=1}^{L} \sum_{n=1}^{N} \sum_{m=1}^{M}\left(\frac{\xi_{\mathrm{F}-\mathrm{M}}^{2} \zeta}{2\left(\xi_{\mathrm{F}-\mathrm{M}}^{2} \zeta+1\right)}\right)^{2}}=\frac{\left(\sum_{l=1}^{L} \sum_{n=1}^{N} \sum_{m=1}^{M} \frac{\xi_{\mathrm{F}-\mathrm{M}}^{2} \zeta}{\xi_{\mathrm{F}-\mathrm{M}}^{2} \zeta+1}\right)^{2}}{\sum_{l=1}^{L} \sum_{n=1}^{N} \sum_{m=1}^{M}\left(\frac{\xi_{\mathrm{F}-\mathrm{M}}^{2} \zeta}{\xi_{\mathrm{F}-\mathrm{M}}^{2} \zeta+1}\right)^{2}} \\
& \beta_{0}^{\mathrm{I}}=\frac{2 \sum_{l=1}^{L} \sum_{n=1}^{N} \sum_{m=1}^{M}\left(\tilde{\kappa}_{n m l}^{\mathrm{F}-\mathrm{M}}\right)^{2}}{\sum_{l=1}^{L} \sum_{n=1}^{N} \sum_{m=1}^{M} \tilde{\kappa}_{n m l}^{\mathrm{F}-\mathrm{M}}}=\frac{2 \sum_{l=1}^{L} \sum_{n=1}^{N} \sum_{m=1}^{M}\left(\frac{\xi_{\mathrm{F}-\mathrm{M}}^{2} \zeta}{2\left(\xi_{\mathrm{F}-\mathrm{M}}^{2} \zeta+1\right)}\right)^{2}}{\sum_{l=1}^{L} \sum_{n=1}^{N} \sum_{m=1}^{M} \frac{\xi_{\mathrm{F}-\mathrm{M}}^{2} \zeta}{2\left(\xi_{\mathrm{F}-\mathrm{M}}^{2} \zeta+1\right)}}=\frac{\sum_{l=1}^{L} \sum_{n=1}^{N} \sum_{m=1}^{M}\left(\frac{\xi_{\mathrm{F}-\mathrm{M}}^{2} \zeta}{\xi_{\mathrm{F}-\mathrm{M}}^{2} \zeta+1}\right)^{2}}{\sum_{l=1}^{L} \sum_{n=1}^{N} \sum_{m=1}^{M} \frac{\xi_{\mathrm{F}-\mathrm{M}}^{2} \zeta}{\xi_{\mathrm{F}-\mathrm{M}}^{2} \zeta+1}} \\
& \alpha_{1}^{\mathrm{I}}=\frac{\left(\sum_{l=1}^{L} \sum_{n=1}^{N} \sum_{m=1}^{M} \tilde{\kappa}_{n m l}^{\mathrm{F}-\mathrm{M}^{\prime}}\right)^{2}}{\sum_{l=1}^{L} \sum_{n=1}^{N} \sum_{m=1}^{M}\left(\tilde{\kappa}_{n m l}^{\mathrm{F}-\mathrm{M}^{\prime}}\right)^{2}}=\frac{\left(\sum_{l=1}^{L} \sum_{n=1}^{N} \sum_{m=1}^{M} \frac{\xi_{\mathrm{F}-\mathrm{M}}^{2} \zeta}{2}\right)^{2}}{\sum_{l=1}^{L} \sum_{n=1}^{N} \sum_{m=1}^{M}\left(\frac{\xi_{\mathrm{F}-\mathrm{M}}^{2} \zeta}{2}\right)^{2}}=\frac{\left(\sum_{l=1}^{L} \sum_{n=1}^{N} \sum_{m=1}^{M} \xi_{\mathrm{F}-\mathrm{M}}^{2}\right)^{2}}{\sum_{l=1}^{L} \sum_{n=1}^{N} \sum_{m=1}^{M}\left(\xi_{\mathrm{F}-\mathrm{M}}^{2}\right)^{2}} \\
& \beta_{1}^{\mathrm{I}}=\frac{2 \sum_{l=1}^{L} \sum_{n=1}^{N} \sum_{m=1}^{M}\left(\tilde{\kappa}_{n m l}^{\mathrm{F}-\mathrm{M}^{\prime}}\right)^{2}}{\sum_{l=1}^{L} \sum_{n=1}^{N} \sum_{m=1}^{M} \tilde{\kappa}_{n m l}^{\mathrm{F}-\mathrm{M}^{\prime}}}=\frac{2 \sum_{l=1}^{L} \sum_{n=1}^{N} \sum_{m=1}^{M}\left(\frac{\xi_{\mathrm{F}-\mathrm{M}}^{2} \zeta}{2}\right)^{2}}{\sum_{l=1}^{L} \sum_{n=1}^{N} \sum_{m=1}^{M} \frac{\xi_{\mathrm{F}-\mathrm{M}}^{2} \zeta}{2}}=\frac{\zeta \sum_{l=1}^{L} \sum_{n=1}^{N} \sum_{m=1}^{M}\left(\xi_{\mathrm{F}-\mathrm{M}}^{2}\right)^{2}}{\sum_{l=1}^{L} \sum_{n=1}^{N} \sum_{m=1}^{M} \xi_{\mathrm{F}-\mathrm{M}}^{2}}
\end{aligned}
$$


$z_{n m l}^{\mathrm{F}-\mathrm{M}}$ is a exponential random variable with the variance $1 / \sigma_{n}^{2}$ under the null hypothesis $H_{0}$. Therefore, we can define a new chi-square random variable with 2 degrees of freedom $\tilde{\Upsilon}_{n m l}^{\mathrm{F}-\mathrm{M}}=\frac{2}{\sigma_{n}^{2}} z_{n m l}^{\mathrm{F}-\mathrm{M}}=2 \Upsilon_{n m l}^{\mathrm{F}-\mathrm{M}}$, where $\tilde{\Upsilon}_{n m l}^{\mathrm{F}-\mathrm{M}} \sim e\left(\frac{1}{2}\right)$. Accordingly, the detection statistic of non-coherent FDA-MIMO radar under the null hypothesis $H_{0}$ can be regarded as the weighted sum of multiple independent and identically distributed (IID) exponential random variables. It follows that $T_{\mathrm{F}-\mathrm{M}}^{\mathrm{I}} \mid H_{0}$ is set using the following formula

$$
T_{\mathrm{F}-\mathrm{M}}^{\mathrm{I}} \mid H_{0}=\sum_{l=1}^{L} \sum_{n=1}^{N} \sum_{m=1}^{M}\left(\tilde{\kappa}_{n m l}^{\mathrm{F}-\mathrm{M}} \tilde{\Upsilon}_{n m l}^{\mathrm{F}-\mathrm{M}}\right) \sim \Gamma\left(\alpha_{0}^{\mathrm{I}}, \beta_{0}^{\mathrm{I}}\right)
$$

where $\tilde{\kappa}_{n m l}^{\mathrm{F}-\mathrm{M}}=\frac{1}{2} \kappa_{n m l}^{\mathrm{F}-\mathrm{M}} . \quad \Gamma\left(\alpha_{0}, \beta_{0}\right)$ represents the PDF of Gamma random process, the shape and scale parameters are $\alpha_{0}$ and $\beta_{0}$, respectively. Moreover, the PDF of Gamma process can be calculated by the formula $f(x, \alpha, \beta)=$ $\frac{\beta^{-\alpha}}{\Gamma(\alpha)} x^{\alpha-1} e^{-\frac{x}{\beta}}, x>0$. In general, the shape and scale parameters $\alpha_{0}^{\mathrm{I}}$ and $\beta_{0}^{\mathrm{I}}$ of non-coherent FDA-MIMO radar can be calculated by (23) and (24) [see previous page], respectively.

Similarly, a new chi-square random variable with 2 degrees of freedom $\tilde{\Upsilon}_{n m l}^{\mathrm{F}-\mathrm{M}^{\prime}}=\frac{2}{\sigma_{n}^{2}\left(\xi_{\mathrm{F}-\mathrm{M}}^{2} \zeta+1\right)} z_{n m l}^{\mathrm{F}-\mathrm{M}}=\frac{2 \Upsilon_{n m l}^{\mathrm{F}-\mathrm{M}}}{\xi_{\mathrm{F}-\mathrm{M}}^{2} \zeta+1}$ is defined under the alternate hypothesis $H_{1}$, where $\tilde{\Upsilon}_{n m l}^{\mathrm{F}-\mathrm{M}^{\prime}} \sim$ $e\left(\frac{1}{2}\right)=\chi_{(2)}^{2}$. It follows that the detection statistics of noncoherent FDA-MIMO radar under the alternate hypothesis $H_{1}$ is set using the following formula

$$
T_{\mathrm{F}-\mathrm{M}}^{\mathrm{I}} \mid H_{1}=\sum_{l=1}^{L} \sum_{n=1}^{N} \sum_{m=1}^{M}\left(\tilde{\kappa}_{n m l}^{\mathrm{F}-\mathrm{M}^{\prime}} \tilde{\Upsilon}_{n m l}^{\mathrm{F}-\mathrm{M}^{\prime}}\right) \sim \Gamma\left(\alpha_{1}^{\mathrm{I}}, \beta_{1}^{\mathrm{I}}\right)
$$

where $\tilde{\kappa}_{n m l}^{\mathrm{F}-\mathrm{M}^{\prime}}=\frac{\xi_{\mathrm{F}-\mathrm{M}}^{2} \zeta+1}{2} \kappa_{n m l}^{\mathrm{F}-\mathrm{M}}=\frac{\xi_{\mathrm{F}-\mathrm{M}}^{2} \zeta}{2}$, are the shape and scale parameters $\alpha_{1}^{\mathrm{I}}$ and $\beta_{1}^{\mathrm{I}}$ that can be represented by (26) and (27), respectively. Generally, this leads to the following distribution of the detection statistics for non-coherent FDAMIMO radar:

$$
T_{\mathrm{F}-\mathrm{M}}^{\mathrm{I}} \sim \begin{cases}\Gamma\left(\alpha_{0}^{\mathrm{I}}, \beta_{0}^{\mathrm{I}}\right) & H_{0} \\ \Gamma\left(\alpha_{1}^{\mathrm{I}}, \beta_{1}^{\mathrm{I}}\right) & H_{1}\end{cases}
$$

where the parameters $\alpha_{0}^{\mathrm{I}}, \beta_{0}^{\mathrm{I}}, \alpha_{1}^{\mathrm{I}}$ and $\beta_{1}^{\mathrm{I}}$ have been defined in (23), (24), (26) and (27) [see previous page], respectively. As for non-coherent FDA-MIMO radar, the probability of detection and false alarm can be expressed as follows:

$$
\begin{aligned}
P_{\mathrm{d}-\mathrm{F}-\mathrm{M}}^{\mathrm{I}}(\eta) & =P\left(T_{\mathrm{F}-\mathrm{M}}^{\mathrm{I}}>\eta \mid H_{1}\right)=P\left(\Gamma\left(\alpha_{1}^{\mathrm{I}}, \beta_{1}^{\mathrm{I}}\right)>\eta\right) \\
& =1-F_{\Gamma\left(\alpha_{1}^{\mathrm{I}}, \beta_{1}^{\mathrm{I}}\right)}(\eta) \\
P_{\mathrm{fa}-\mathrm{F}-\mathrm{M}}^{\mathrm{I}}(\eta) & =P\left(T_{\mathrm{F}-\mathrm{M}}^{\mathrm{I}}>\eta \mid H_{0}\right)=P\left(\Gamma\left(\alpha_{0}^{\mathrm{I}}, \beta_{0}^{\mathrm{I}}\right)>\eta\right) \\
& =1-F_{\Gamma\left(\alpha_{0}^{\mathrm{I}}, \beta_{0}^{\mathrm{I}}\right)}(\eta)
\end{aligned}
$$

where $F_{\Gamma\left(\alpha_{i}, \beta_{i}\right)}(\cdot)(i=0,1)$ represents the cumulative distribution function (CDF) of Gamma random process $\Gamma\left(\alpha_{i}, \beta_{i}\right)(i=$ $0,1)$. It follows that the detection threshold $\eta_{\mathrm{F}-\mathrm{M}}^{\mathrm{I}}$ is set using the following formula

$$
\eta_{\mathrm{F}-\mathrm{M}}^{\mathrm{I}}\left(P_{\mathrm{f}_{0}}\right)=F_{\Gamma\left(\alpha_{0}^{\mathrm{I}}, \beta_{0}^{\mathrm{I}}\right)}^{-1}\left(1-P_{\mathrm{f}_{0}}\right)
$$

where $P_{\mathrm{f}_{0}}$ denotes the given the probability of false alarm, and $F_{\Gamma\left(\alpha_{i}, \beta_{i}\right)}^{-1}(\cdot)(i=0,1)$ represents the inverse CDF of Gamma random process $\Gamma\left(\alpha_{i}, \beta_{i}\right)(i=0,1)$. The probability of detection is given by

$$
\begin{aligned}
P_{\mathrm{d}-\mathrm{F}-\mathrm{M}}^{\mathrm{I}}\left(P_{\mathrm{f}_{0}}\right) & =1-F_{\Gamma\left(\alpha_{1}^{\mathrm{I}}, \beta_{1}^{\mathrm{I}}\right)}\left(\eta_{\mathrm{F}-\mathrm{M}}^{\mathrm{I}}\left(P_{\mathrm{f}_{0}}\right)\right) \\
& =1-F_{\Gamma\left(\alpha_{1}^{\mathrm{I}}, \beta_{1}^{\mathrm{I}}\right)}\left(F_{\Gamma\left(\alpha_{0}^{\mathrm{I}}, \beta_{0}^{\mathrm{I}}\right)}^{-1}\left(1-P_{\mathrm{f}_{0}}\right)\right) .
\end{aligned}
$$




\subsection{Coherent FDA-MIMO Radar}

When using co-located antennas, the reflection coefficients of coherent FDA-MIMO radar can be simplified to coherent model, and the attenuation factor $\delta_{n m}$ for the $n m$ th channel would degenerate into a constant $\delta_{0}$. Similar with Sec. 3.2, the detection statistics of coherent FDA-MIMO radar can be simply derived as follows.

Under the null hypothesis $H_{0}$, the variable $\tilde{\kappa}_{n m l}^{\mathrm{F}}=\frac{1}{2} \kappa_{n m l}^{\mathrm{F}}$ can be redefined as

$$
\tilde{\kappa}_{n m l}^{\mathrm{F}}=\frac{\delta_{0}^{2} \delta_{\varphi \theta}^{2} \delta_{\Delta f}^{2} \zeta}{\delta_{0}^{2} \delta_{\varphi \theta}^{2} \delta_{\Delta f}^{2} \zeta+1}=\frac{\xi_{\mathrm{F}}^{2} \zeta}{\xi_{\mathrm{F}}^{2} \zeta+1}
$$

where $\xi_{\mathrm{F}}^{2}=\delta_{0}^{2} \delta_{\varphi \theta}^{2} \delta_{\Delta f}^{2}$ is the unique parameter of coherent FDA-MIMO radar. Similarly, the shape and scale parameters $\alpha_{0}^{\mathrm{C}}$ and $\beta_{0}^{\mathrm{C}}$ of coherent FDA-MIMO radar can be calculated by (34) and (35) [see previous page] respectively.

Accordingly, the shape and scale parameters $\alpha_{1}^{\mathrm{C}}$ and $\beta_{1}^{\mathrm{C}}$ of coherent FDA-MIMO radar under the alternate hypothesis $H_{1}$ can be calculated by (36) and (37) [see previous page], respectively. This leads to the following distribution of the detection statistics of coherent FDA-MIMO radar

$$
T_{\mathrm{F}}^{\mathrm{C}} \sim\left\{\begin{array}{cc}
\frac{\xi_{\mathrm{F}}^{2} \zeta}{2\left(\xi_{\mathrm{F}}^{2} \zeta+1\right)} \chi_{(2 \mathrm{NML})}^{2} & H_{0} \\
\frac{\xi_{\mathrm{F}}^{2} \zeta}{2} \chi_{(2 \mathrm{NML})}^{2} & H_{1}
\end{array}\right.
$$

where the parameters $\alpha_{0}^{\mathrm{C}}, \beta_{0}^{\mathrm{C}}, \alpha_{1}^{\mathrm{C}}$ and $\beta_{1}^{\mathrm{C}}$ have been defined in (34), (35), (36) and (37) [see previous page], respectively. Similarly, the probability of detection and false alarm of coherent FDA-MIMO radar can be expressed as follows

$$
\begin{aligned}
P_{\mathrm{d}-\mathrm{F}}^{\mathrm{C}}(\eta) & =P\left(T_{\mathrm{F}}^{\mathrm{C}}>\eta \mid H_{1}\right)=P\left(\frac{\xi_{\mathrm{F}}^{2} \zeta}{2} \chi_{(2 \mathrm{NML})}^{2}>\eta\right) \\
& =1-F_{\chi_{(2 \mathrm{NML})}^{2}}\left(\frac{2 \eta}{\xi_{\mathrm{F}}^{2} \zeta}\right), \\
P_{\mathrm{fa}-\mathrm{F}}^{\mathrm{C}}(\eta)= & P\left(T_{\mathrm{F}}^{\mathrm{C}}>\eta \mid H_{0}\right)=P\left(\frac{\xi_{\mathrm{F}}^{2} \zeta}{2\left(\xi_{\mathrm{F}}^{2} \zeta+1\right)} \chi_{(2 \mathrm{NML})}^{2}>\eta\right) \\
= & 1-F_{\chi_{(2 \mathrm{NML})}^{2}}\left(\frac{2 \eta\left(\xi_{\mathrm{F}}^{2} \zeta+1\right)}{\xi_{\mathrm{F}}^{2} \zeta}\right)
\end{aligned}
$$

where $F_{\chi_{(2 \mathrm{NML})}^{2}}(\cdot)$ represents the CDF of Chi-square function $\chi_{(2 \mathrm{NML})}^{2}$. It follows that the detection threshold $\eta_{\mathrm{F}}^{\mathrm{C}}$ is set using the following formula

$$
\eta_{\mathrm{F}}^{\mathrm{C}}\left(P_{\mathrm{f}_{0}}\right)=\frac{\xi_{\mathrm{F}}^{2} \zeta}{2\left(\xi_{\mathrm{F}}^{2} \zeta+1\right)} F_{\chi_{(2 \mathrm{NML})}^{2}}^{-1}\left(1-P_{\mathrm{f}_{0}}\right)
$$

where $F_{\chi_{(2 \mathrm{NML})}^{2}}^{-1}(\cdot)$ represents the inverse CDF of Chi-square function $\chi_{(2 \mathrm{NML})}^{2}$. The probability of detection is given by

$$
\begin{aligned}
P_{\mathrm{d}-\mathrm{F}}^{\mathrm{C}}\left(P_{\mathrm{f}_{0}}\right) & =1-F_{\chi_{(2 \mathrm{NML})}^{2}}\left(\frac{2 \eta_{\mathrm{F}}^{\mathrm{C}}\left(P_{\mathrm{f}_{0}}\right)}{\xi_{\mathrm{F}}^{2} \zeta}\right) \\
& =1-F_{\chi_{(2 \mathrm{NML})}^{2}}\left(\frac{1}{\xi_{\mathrm{F}}^{2} \zeta+1} F_{\chi_{(2 \mathrm{NML})}^{2}}^{-1}\left(1-P_{\mathrm{f}_{0}}\right)\right) .
\end{aligned}
$$

\begin{tabular}{|c|c|c|}
\hline Symbol & Parameter & Value \\
\hline$f_{0}$ & Reference frequency & $10 \mathrm{GHz}$ \\
\hline$\Delta f$ & Frequency increment & $30 \mathrm{kHz}$ \\
\hline$H$ & Platform height & $3000 \mathrm{~m}$ \\
\hline$v_{\mathrm{p}}$ & Platform velocity & $90 \mathrm{~m} / \mathrm{s}$ \\
\hline$M$ & Transmitting elements & 4,8 \\
\hline$N$ & Receiving elements & 4,8 \\
\hline$f_{\text {prf }}$ & PRF & $15 \mathrm{kHz}$ \\
\hline$L$ & Number of pulses & 4 \\
\hline$d$ & Element spacing & $0.0075 \mathrm{~m}$ \\
\hline
\end{tabular}

Tab. 2. Parameters of airborne FDA radar.

\subsection{PA and MIMO Radar}

In addition, the closed-form formulas to show the relationship between probability of detection and false alarm of PA and MIMO radars can be expressed as follows [29]

$$
\begin{aligned}
& P_{\mathrm{d}-\mathrm{P}}\left(P_{\mathrm{f}_{0}}\right)=1-F_{\chi_{(2 \mathrm{~L})}^{2}}\left(\frac{1}{M^{2} N \zeta+1} F_{\chi(2 \mathrm{~L})}^{-1}\left(1-P_{\mathrm{f}_{0}}\right)\right), \\
& P_{\mathrm{d}-\mathrm{M}}\left(P_{\mathrm{f}_{0}}\right)=1-F_{\chi_{(2 \mathrm{NML})}^{2}}\left(\frac{1}{\xi_{\mathrm{M}}^{2} \zeta+1} F_{\chi_{(2 \mathrm{NML})}^{2}}^{-1}\left(1-P_{\mathrm{f}_{0}}\right)\right)
\end{aligned}
$$

where $\xi_{\mathrm{M}}^{2}=\delta_{0}^{2} \delta_{\varphi \theta}^{2}$ is the unique parameter of airborne MIMO radar.

\section{Numerical Results}

We compare the detection performance of the coherent FDA-MIMO (Co-FM) and non-coherent FDA-MIMO (NoFM) radars with that of the conventional PA and MIMO radars. Unless stated otherwise, the simulation parameters listed in Tab. 2 are used in all the experiments.

\subsection{Detection Performance versus SNR}

Figure 3 shows the detection performance under sidelooking operation for a single target. This experiment mainly analyses the array structures influence on the detection performance. It is noticed that coherent FDA-MIMO radar can achieve better detection performance than PA radar at high SNR. Compared with MIMO radar, the performance of non-coherent FDA-MIMO radar is significantly improved at low SNR. Increasing the number of transmitting array elements will greatly improve the detection performance of FDA-MIMO radars.

As shown in Fig. 3(a), the performance of PA is better than non-coherent FDA-MIMO radar at low SNR, and the detection probability of the coherent FDA-MIMO and MIMO radars are almost equal to 0 . When the SNR is greater than $-5 \mathrm{~dB}$, the detection probability of coherent FDA-MIMO and MIMO radars increases rapidly. The detection performance of all array structures improve according to the increasing number of receive elements. At low SNR, the performance improvement for PA radar is largest, while that of 
FDA-MIMO and MIMO radars. Shown in Fig. 3(b), the detection probability of FDA-MIMO is significantly enhanced with an increasing number of transmit elements, whereas the improvement of coherent and non-coherent FDA-MIMO are more can $10 \mathrm{~dB}$. In this case, the performance of coherent FDA-MIMO radar is better than that of non-coherent FDAMIMO radar at low SNR, but the results become inverse with high SNR.

\begin{tabular}{|c|c|c|c|c|c|}
\hline Parameter & SNR [dB] & -12 & -3 & 0 & 3 \\
\hline \multirow{3}{*}{$\begin{array}{l}M=4 \\
L=1\end{array}$} & PA & 0.1018 & 0.7060 & 0.8377 & 0.9144 \\
\cline { 2 - 6 } & MIMO & $4.21 \mathrm{e}-5$ & 0.0141 & 0.1826 & 0.7604 \\
\cline { 2 - 6 } & Co-FM & $8.23 \mathrm{e}-5$ & 0.0736 & 0.5224 & 0.9625 \\
\cline { 2 - 6 } & No-FM & $3.56 \mathrm{e}-4$ & 0.2729 & 0.5070 & 0.6817 \\
\hline \multirow{3}{*}{$\begin{array}{l}M=4 \\
L=1\end{array}$} & PA & 0.2813 & 0.8380 & 0.9146 & 0.9561 \\
\cline { 2 - 6 } & MIMO & $6.32 \mathrm{e}-5$ & 0.0582 & 0.5457 & 0.9872 \\
\cline { 2 - 6 } & Co-FM & $1.48 \mathrm{e}-4$ & 0.2754 & 0.9156 & 0.9999 \\
\cline { 2 - 6 } & No-FM & 0.0014 & 0.4388 & 0.6316 & 0.7592 \\
\hline \multirow{3}{*}{$\begin{array}{l}M=8 \\
L=1\end{array}$} & PA & 0.5111 & 0.9148 & 0.9562 & 0.9778 \\
\cline { 2 - 6 } & MIMO & $6.32 \mathrm{e}-5$ & 0.0582 & 0.5457 & 0.9872 \\
\cline { 2 - 6 } & Co-FM & 0.1690 & 0.9999 & 1.0000 & 1.0000 \\
\cline { 2 - 6 } & No-FM & 0.3873 & 0.8313 & 0.8976 & 0.9411 \\
\hline \multirow{3}{*}{$\begin{array}{l}M=4 \\
L=4\end{array}$} & PA & 0.4931 & 0.9973 & 0.9998 & 1.0000 \\
\cline { 2 - 6 } & MIMO & $1.11 \mathrm{e}-4$ & 0.2471 & 0.9410 & 1.0000 \\
\cline { 2 - 6 } & Co-FM & $3.27 \mathrm{e}-4$ & 0.7304 & 0.9994 & 1.0000 \\
\cline { 2 - 6 } & No-FM & 0.0069 & 0.5753 & 0.7154 & 0.8082 \\
\hline
\end{tabular}

Tab. 3. Detection probability of array radars in different scenarios.

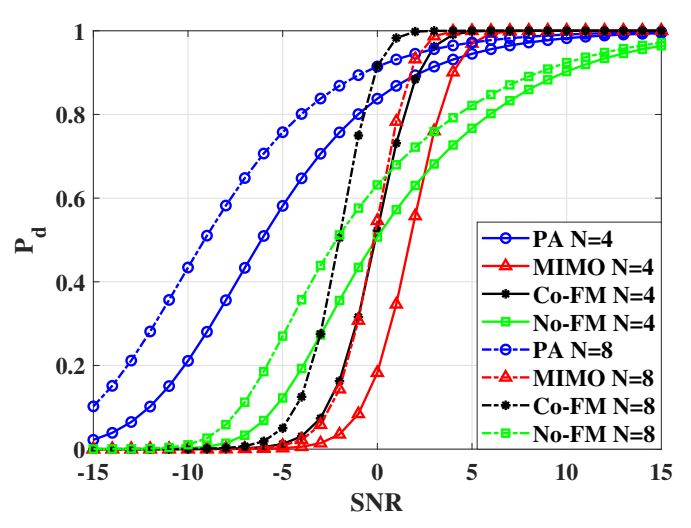

(a) $M=4, L=1$

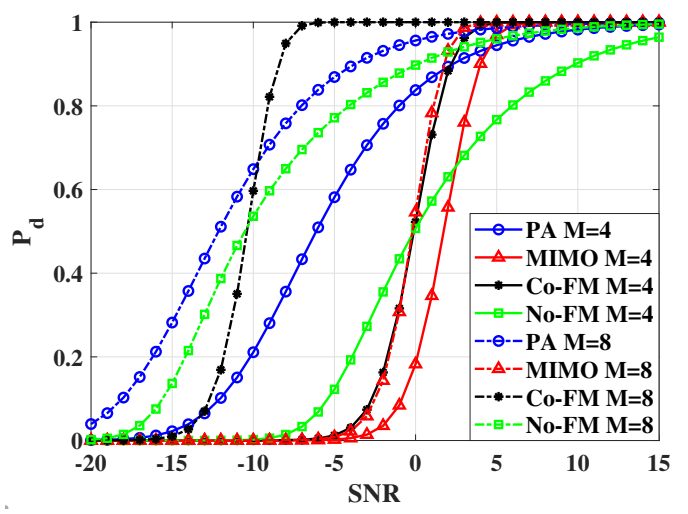

(b) $N=4, L=1$

Fig. 3. Detection performance versus SNR of airborne array radars with different number of elements.
Figure 4 shows the detection performance of PA, MIMO and FDA-MIMO radars having the number of transmit and receive elements $M=8$ and $N=4$, respectively. The detection performance of all array structures improve according to the increased number of pulses. The improvement of PA radar is significant, while that of FDA-MIMO and MIMO radars are slightly improved. When SNR is less than $-10 \mathrm{~dB}$, the performance of PA radar is better than that of non-coherent FDA-MIMO radars. However, the detection probability of coherent FDA-MIMO radar rapidly increases than others when the SNR is greater than $-10 \mathrm{~dB}$. In addition, the detection probabilities corresponding to several SNRs of different array structures are summarized in Tab. 3 .

Figure 5 shows the influence of the frequency increments $\Delta f$ on detection probability. When the frequency increment is set as $\Delta f=10 \mathrm{kHz}$, the detection performance of non-coherent FDA-MIMO is better than coherent FDA-MIMO radar at low SNR. Moreover, when SNR is greater than $-5 \mathrm{~dB}$, the detection probability of coherent FDA-MIMO and MIMO radars rapidly improves. Additionally, the performance of FDA-MIMO radars significantly approach PA with $\Delta f=30 \mathrm{kHz}$. Therefore, selecting an appropriate frequency increment will effectively enhance the detection performance of coherent and noncoherent FDA-MIMO radars.

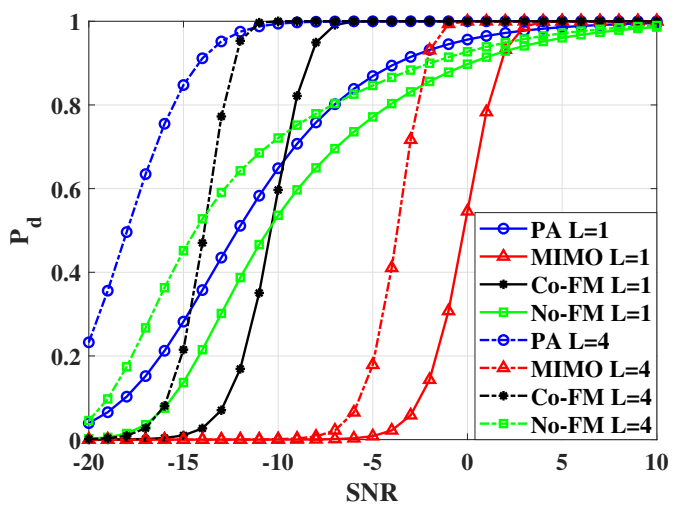

Fig. 4. Detection performance versus SNR of airborne array radars with different number of pulses.

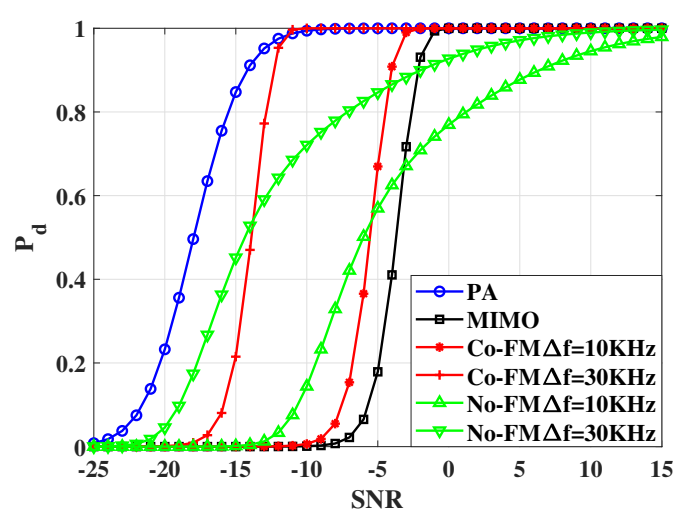

Fig. 5. Detection performance versus SNR of airborne FDAMIMO radar with different $\Delta f$. 


\subsection{Detection Performance versus False Alarm Probability $\left(\mathbf{P}_{\mathbf{f}}\right)$}

Figure 6 shows the array structures influence on the detection performance. Fig. 6(a) and (b) establish the advantages of the coherent FDA-MIMO over PA and MIMO radars. It is noticed that coherent FDA-MIMO radar can achieve better detection performance than PA, MIMO and non-coherent FDA-MIMO radars with large number of transmit elements.

It is shown in Fig. 6(a) that the coherent FDA-MIMO outperforms the PA, MIMO and non-coherent FDA-MIMO radars with $M=4$ and $M=8$. Obviously, when the false alarm probability $\left(\mathrm{P}_{\mathrm{f}}\right)$ is greater than $10^{-6}$, the performance of MIMO is better than PA radar, whereas, that of non-coherent FDA-MIMO is worst at $\mathrm{SNR}=5 \mathrm{~dB}$. Figure $6(\mathrm{~b})$ shows that coherent FDA-MIMO can achieve best performance with $\mathrm{SNR}=-5 \mathrm{~dB}$, and the non-coherent FDA-MIMO offers a compromise between PA and MIMO radars. The increased number of transmit array elements will significantly enhance the detection performance of FDA-MIMO radars.

Figure 7 shows the influence of frequency increment $\Delta f$ on detection performance. Figure 7(a) and (b) analyse the advantages of FDA-MIMO radars with different $\Delta f$, and validate that both coherent and non-coherent FDA-MIMO radars outperform the PA and MIMO radars with appropriate frequency increment.

Figure 7(a) shows that the coherent FDA-MIMO outperforms other radar designs with both of the frequency increment i.e., $\Delta f=30 \mathrm{kHz}$ and $\Delta f=10 \mathrm{kHz}$. Moreover, the detection probability of non-coherent FDA-MIMO radar with $\Delta f=10 \mathrm{kHz}$ is greater than $\mathrm{PA}$ and MIMO radars when $\mathrm{P}_{\mathrm{f}}$ is smaller than $10^{-6}$. Obviously, when $\Delta f=10 \mathrm{kHz}$, the FDA-MIMO radars outperform the PA and MIMO radars at $\mathrm{SNR}=-5 \mathrm{~dB}$. Additionally, when $\Delta f=30 \mathrm{kHz}$, the performance of both coherent and non-coherent FDA-MIMO radars significantly approach MIMO in this case.

\subsection{Detection Performance versus Azimuth $\theta$}

Assuming the azimuth of target near $0^{\circ}$, Figure 8 shows the detection performance of PA, MIMO and FDA-MIMO radars with $\mathrm{SNR}=3 \mathrm{~dB}$. It is shown that when $M=4$, the non-coherent FDA-MIMO significantly outperforms other designs, while the performance of PA compromises with MIMO and coherent FDA-MIMO radars. However, the detection probability of all array structures approach to 1 with the number of transmit elements increasing to 8 .

Figure 9 shows the detection performance of PA, MIMO and FDA-MIMO radars with different SNR. When SNR is $3 \mathrm{~dB}$, the performance of PA radar is best, while the coherent FDA-MIMO design compromises with MIMO and non-coherent FDA-MIMO radars. When SNR is decreased to $-3 \mathrm{~dB}$, the performance of coherent FDA-MIMO and MIMO radars drastically decreases, while performance of the non-coherent FDA-MIMO and PA radars remains maintained.

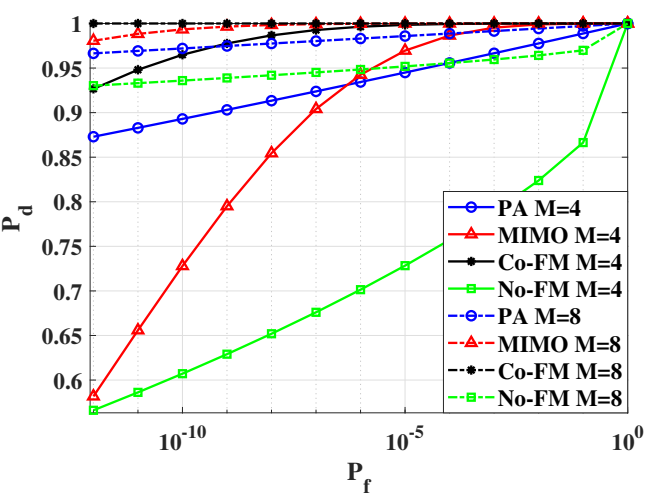

(a) $\mathrm{SNR}=5 \mathrm{~dB}$

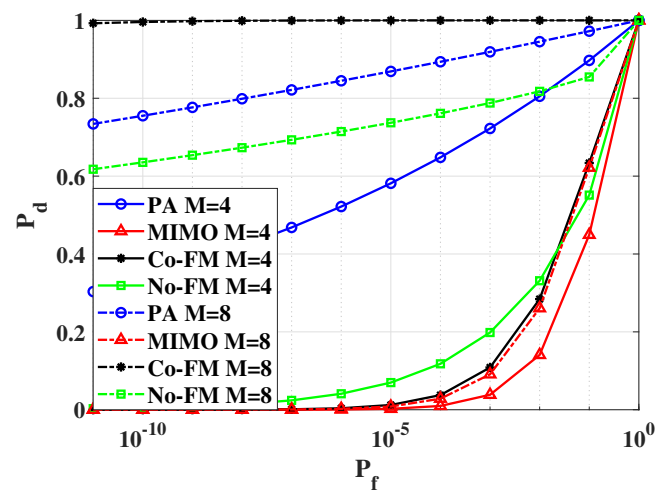

(b) $\mathrm{SNR}=-5 \mathrm{~dB}$

Fig. 6. Detection performance versus $P_{f}$ of airborne array radar with different number of transmit elements

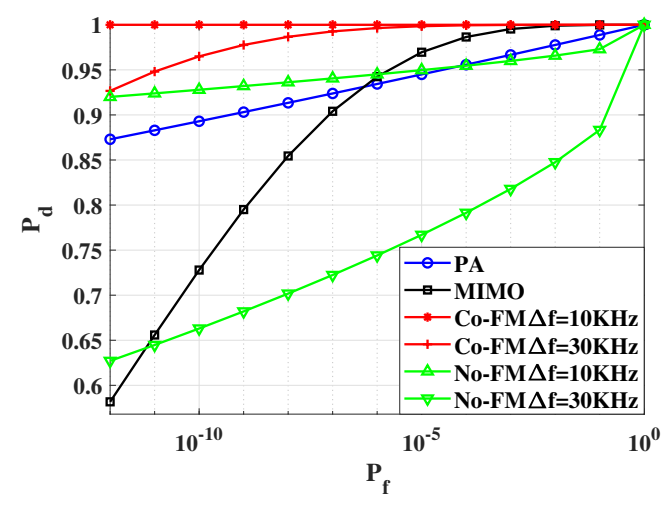

(a) $\mathrm{SNR}=5 \mathrm{~dB}$

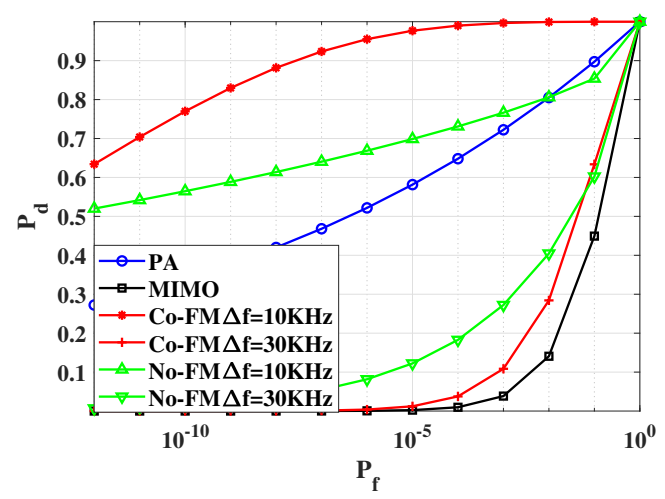

(b) $\mathrm{SNR}=-5 \mathrm{~dB}$

Fig. 7. Detection performance versus $\mathrm{P}_{\mathrm{f}}$ of airborne FDAMIMO radar with different $\Delta f$. 


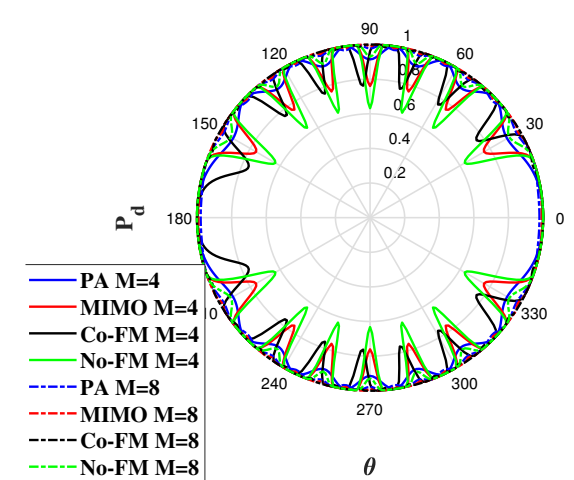

Fig. 8. Isoprobability curve with different number of transmit elements.

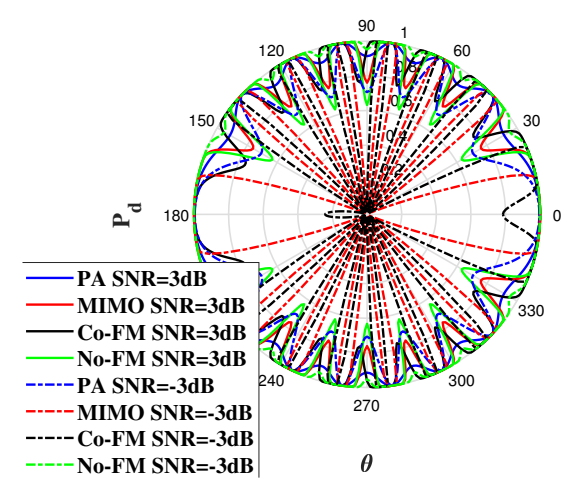

Fig. 9. Isoprobability curve with different SNR.

\subsection{Detection Performance versus Different Ranges}

For the airborne array radars, the range of target can be represented by its elevation angles $\varphi$. Figure 10(a), (c), (e), (g) [see next page] compare the detection performance of PA, MIMO, coherent FDA-MIMO and non-coherent FDA-MIMO radars in different range bins with $\mathrm{SNR}=3 \mathrm{~dB}$. The detection probability of PA radar in all ranges performs almost the same, which cannot distinguish the targets in different ranges. The isoprobability curves with MIMO and coherent FDA-MIMO radar are shown in Fig. 10(c) and (e), the detection probability of these are changing with the varying ranges that can be used to distinguish the range-dependent targets. Obviously, the sensitivity of non-coherent FDA-MIMO radar to ranges achieve the better detection performance with the existence of rangedependent targets.

As shown in Fig. 10(b), (d), (f), (h) [see next page], the detection performance of MIMO, coherent and non-coherent FDA-MIMO radars degrade drastically. It is noticed that only PA radar can maintain good detection performance with decreasing SNR. Consequently, the range dependent targets cannot be distinguished by the existing detection algorithms of FDA-MIMO radars at low SNR.

\section{Conclusion}

A theoretical study on the detection performance of the airborne coherent and non-coherent FDA-MIMO radars with incoherent square-law detector have been carried out. It has been validated that the promising capability of FDA-MIMO radars for improved detection probability can be controlled by exploring the extra DOFs due to the frequency increment. Moreover, non-coherent FDA-MIMO radar can distinguish the range-dependent targets more effectively than the PA, MIMO, and coherent FDA-MIMO radars. It is necessary to note that the coherent FDA-MIMO and MIMO radars achieve better detection performance than PA and non-coherent FDAMIMO radar at high SNR, but the improvement of coherent FDA-MIMO radar is significant. As the number of transmit elements and pulses increase, the performance of coherent FDA-MIMO becomes much better than MIMO radar, while the non-coherent FDA-MIMO gradually approach to PA radar. Both theoretical analysis and simulation results verify the superiority of FDA-MIMO radars over traditional PA and MIMO radars in target detection. In this paper, we have analyzed the theoretical performance of FDA-MIMO radars to validate its superiorities with IID reflection coefficients. In future work, we plan to further investigate the moving target detection using FDA-MIMO radars with non-IID reflection coefficients.

\section{References}

[1] WARD, J. Space-time adaptive processing for airborne radar. In International Conference on Acoustics, Speech, and Signal Processing. Detroit (USA), 1995, p. 2809-2812. DOI: 10.1109/ICASSP.1995.479429

[2] KLEMM, R. Space-time adaptive processing: principles and applications [book review]. Electronics Communication Engineering Journal, 1999, vol. 11, no. 4, p. 172-172. DOI: 10.1049/ecej:19990404

[3] GUERCI, J. R. Theory and application of covariance matrix tapers for robust adaptive beamforming. IEEE Transactions on Signal Processing, 1999, vol. 47, no. 4, p. 977-985. DOI: 10.1109/78.752596

[4] SWINDLEHURST, A. L., STOICA, P. Maximum likelihood methods in radar array signal processing. Proceedings of the IEEE, 1998, vol. 86, no. 2, p. 421-441. DOI: $10.1109 / 5.659495$

[5] FISHLER, E., HAIMOVICH, A., BLUM, R., et al. Mimo radar: An idea whose time has come. In Proceedings of the IEEE National Radar Conference. Philadelphia (USA), 2004, p. 71-78. DOI: $10.1109 /$ NRC.2004.1316398

[6] FISHLER, E., HAIMOVICH, A., BLUM, R., et al. Performance of MIMO radar systems: Advantages of angular diversity. In Conference Record of the Thirty-Eighth Asilomar Conference on Signals, Systems and Computers. Pacific Grove (USA), 2004, p. 305-309. DOI: 10.1109/ACSSC.2004.1399142

[7] BEKKERMAN, I., TABRIKIAN, J. Spatially coded signal model for active arrays. In IEEE International Conference on Acoustics, Speech, and Signal Processing (ICASSP). Montreal (Canada), 2004, p. 209-212. DOI: 10.1109/ICASSP.2004.1326231

[8] HAIMOVICH, A. M., BLUM, R. S., CIMINI, L. J. Mimo radar with widely separated antennas. IEEE Signal Processing Magazine, 2008, vol. 25, no. 1, p. 116-129. DOI: 10.1109/MSP.2008.4408448 


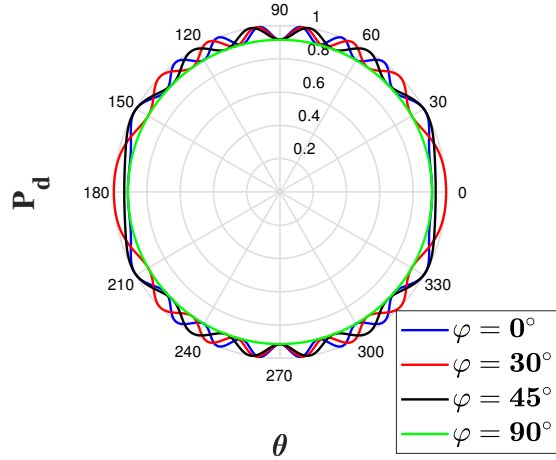

(a)



(c)



(e)

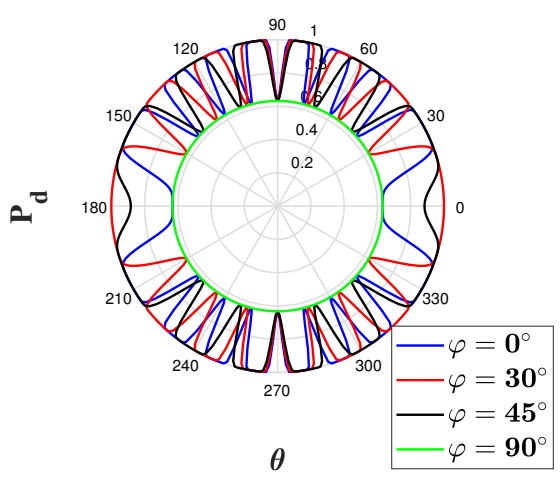

(g)

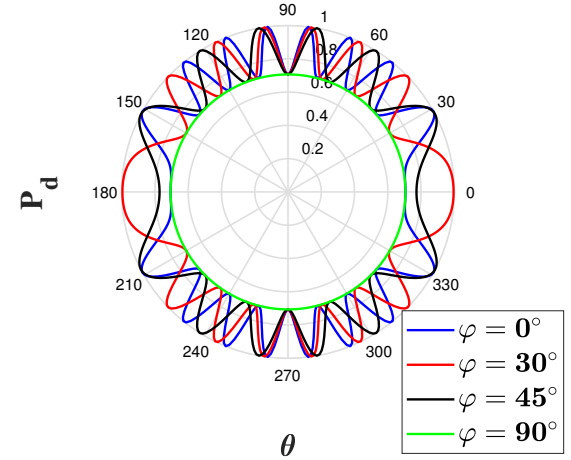

(b)

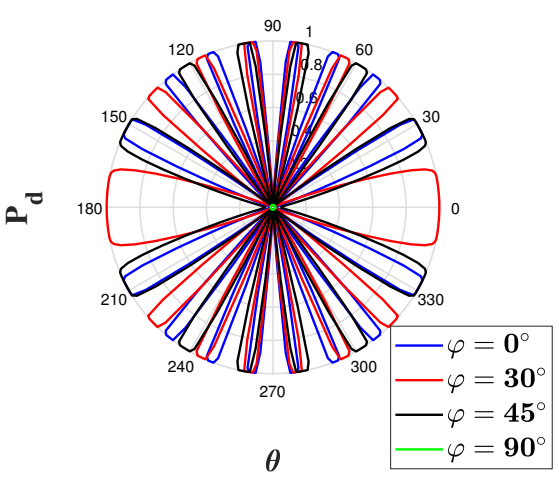

(d)

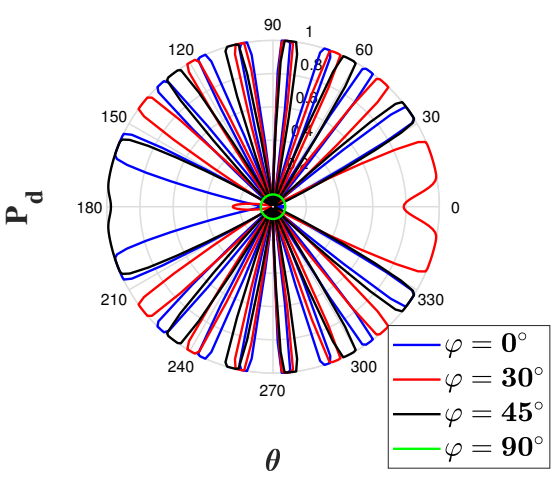

(f)

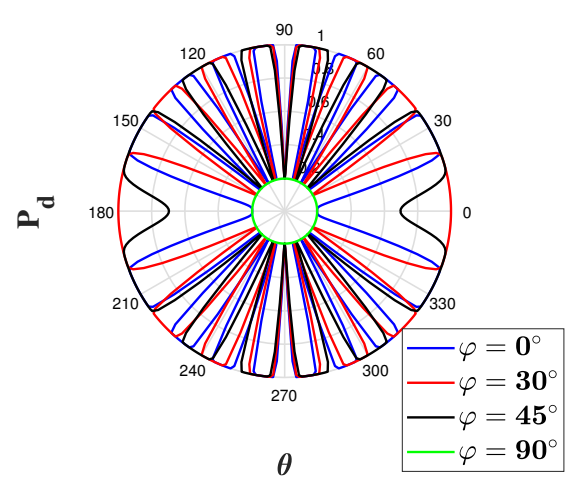

(h)

Fig. 10. Isoprobability curve with different ranges in $3 \mathrm{~dB}$ and $-3 \mathrm{~dB}$. (a) $\mathrm{PA}$ in $3 \mathrm{~dB}$, (b) PA in $-3 \mathrm{~dB}$, (c) MIMO in $3 \mathrm{~dB}$, (d) $\mathrm{MIMO}$ in $-3 \mathrm{~dB}$, (e) coherent FDA-MIMO in $3 \mathrm{~dB}$, (f) coherent FDA-MIMO in - $3 \mathrm{~dB}$, (g) non-coherent FDA-MIMO in $3 \mathrm{~dB}$, (h) non-coherent FDA-MIMO in $-3 \mathrm{~dB}$. 
[9] XU, J., LIAO, G., HUANG, L., et al. Robust adaptive beamforming for fast-moving target detection with FDA-STAP radar. IEEE Transactions on Signal Processing, 2017, vol. 65, no. 4, p. 973-984. DOI: $10.1109 /$ tsp.2016.2628340

[10] BEKKERMAN, I., TABRIKIAN, J. Target detection and localization using mimo radars and sonars. IEEE Transactions on Signal Processing, 2006, vol. 54, no. 10, p. 3873-3883. DOI: $10.1109 /$ TSP.2006.879267

[11] ANTONIK, P., WICKS, M. C., GRIFFITHS, H. D., et al. Frequency diverse array radars. In Proceedings of the IEEE Radar Conference. Verona (USA), 2006, p. 215-217. DOI: 10.1109/RADAR.2006.1631800

[12] WICKS, M. C., ANTONIK, P. Frequency Diverse Array with Independent Modulation of Frequency, Amplitude, and Phase. U.S.A Patent no. US7319427B2, January 15, 2008.

[13] SECMEN, M., DEMIR, S., HIZAL, A., et al. Frequency diverse array antenna with periodic time modulated pattern in range and angle. In Proceedings of the IEEE Radar Conference. Waltham (USA), 2007, p. 427-430. DOI: 10.1109/RADAR.2007.374254

[14] WANG, W.-Q., SO, H. C., FARINA, A. An overview on time/frequency modulated array processing. IEEE Journal of Selected Topics in Signal Processing, 2017, vol. 11, no. 2, p. 228-246. DOI: 10.1109/JSTSP.2016.2627182

[15] WANG, W.-Q., SHAO, H. Z. A flexible phased-MIMO array antenna with transmit beamforming. International Journal of Antennas and Propagation, 2012, vol. 2012, p. 1-10. DOI: 10.1155/2012/609598

[16] ABDALlA, A., WANG, W.-Q., ZHAO, Y., et al. Subarray-based FDA radar to counteract deceptive ECM signals. EURASIP Journal on Advances in Signal Processing, 2016, vol. 2016, no. 1, p. 1-11. DOI: 10.1186/s13634-016-0403-6

[17] WANG, W.-Q., SO, H. C. Transmit subaperturing for range and angle estimation in frequency diverse array radar. IEEE Transactions on Signal Processing, 2014, vol. 62, no. 8, p. 2000-2011. DOI: $10.1109 /$ TSP.2014.2305638

[18] XU, J., ZHU, S., LIAO, G. Range ambiguous clutter suppression for airborne FDA-STAP radar. IEEE Journal of Selected Topics in Signal Processing, 2015, vol. 9, no. 8, p. 1620-1631. DOI: 10.1109/JSTSP.2015.2465353
[19] LAN, L., LIAO, G., XU, J., et al. Suppression approach to mainbeam deceptive jamming in FDA-MIMO radar using nonhomogeneous sample detection. IEEE Access, 2018, vol. 6, p. 34582-34597. DOI: 10.1109/ACCESS.2018.2850816

[20] XU, J., LIAO, G., ZHANG, Y., et al. An adaptive range-angle-doppler processing approach for fda-mimo radar using three-dimensional localization. IEEE Journal of Selected Topics in Signal Processing, 2017, vol. 11, no. 2, p. 309-320. DOI: 10.1109/JSTSP.2016.2615269

[21] WEN, C., TAO, M., PENG, J., et al. Clutter suppression for airborne FDA-MIMO radar using multi-waveform adaptive processing and auxiliary channel STAP. Signal Processing, 2019, vol. 154, p. 280-293. DOI: 10.1016/j.sigpro.2018.09.016

[22] GUI, R., WANG, W., SHAO, H. General receiver design for FDA radar. In IEEE Radar Conference (RadarConf). Oklahoma City (USA), 2018, p. 280-285. DOI: 10.1109/RADAR.2018.8378571

[23] GUI, R., WANG, W., CUI, C., et al. Coherent pulsed-FDA radar receiver design with time-variance consideration: SINR and CRB analysis. IEEE Transactions on Signal Processing, 2018, vol. 66, no. 1, p. 200-214. DOI: 10.1109/TSP.2017.2764860

[24] ZHU, Y., LIU, L., LU, Z., et al. Target detection performance analysis of FDA-MIMO radar. IEEE Access, 2019, vol. 7, p. 164276-164285. DOI: 10.1109/ACCESS.2019.2943082

[25] GUI, R., WANG, W.-Q., ZHENG, Z. Low-complexity GLRT for FDA radar without training data. Digital Signal Processing, 2020, vol. 107, p. 1-11. DOI: 10.1016/j.dsp.2020.102861

[26] LAN, L., MARINO, A., AUBRY, A., et al. GLRT-based adaptive target detection in FDA-MIMO radar. IEEE Transactions on Aerospace and Electronic Systems, 2020, vol. 57, no. 1, p. 597-613. DOI: 10.1109/TAES.2020.3028485

[27] YAN, Y., WANG, W.-Q., ZHANG, S., et al. Range-ambiguous clutter characteristics in airborne FDA radar. Signal Processing, 2019, vol. 170. DOI: 10.1016/j.sigpro.2019.107407

[28] SKOLNIK, M. Introduction to Radar Systems. New York (USA): McGrow-Hill, 2001. ISBN: 9780070579057

[29] FISHLER, E., HAIMOVICH, A., BLUM, R. S., et al. Spatial diversity in radars-models and detection performance. IEEE Transactions on Signal Processing, 2006, vol. 54, no. 3, p. 823-838. DOI: $10.1109 /$ TSP.2005.862813 